\title{
Tumor necrosis factor receptor associated factor-4: An adapter protein overexpressed in metastatic prostate cancer is regulated by microRNA-29a
}

\author{
FOZIA AHMED $^{1,3}$, TAKUMI SHIRAISHI ${ }^{1,4}$, ROBERT L. VESSELLA ${ }^{5}$ and PRAKASH KULKARNI ${ }^{1,2}$ \\ ${ }^{1}$ James Buchanan Brady Urological Institute, Department of Urology, and ${ }^{2}$ Department of Oncology, \\ The Johns Hopkins School of Medicine, Baltimore, MD 21287; ${ }^{3}$ The Johns Hopkins University Krieger School of Arts and \\ Sciences, Baltimore, MD 21218, USA; ${ }^{4}$ Department of Urology, Graduate School of Medical Science, Kyoto Prefectural \\ University of Medicine, Kyoto, Japan; ${ }^{5}$ Department of Urology, University of Washington, Seattle, WA 98104, USA
}

Received August 15, 2013; Accepted September 26, 2013

DOI: 10.3892/or.2013.2789

\begin{abstract}
The tumor necrosis factor receptor (TNFR)associated factor 4 (TRAF4) is a member of TRAF family proteins that act as major signal transducers of the TNF receptor and the interleukin-1 receptor/Toll-like receptor (IL-1R/TLR) superfamily. TRAF4 has been reported to be overexpressed in various human cancers. However, the exact mechanisms that regulate the expression of TRAF4 still remain elusive. The objective of the present study was to investigate the regulatory mechanism of TRAF4 expression in prostate cancer. We initially identified microRNA-29a (miR-29a) as a possible candidate to bind TRAF4 3' untranslated region (3'UTR) by the algorithm, TargetScan. The expression of TRAF4 mRNA and protein was inversely associated with miR-29a expression in prostate cancer cell lines (LNCaP, DU145 and PC3). TRAF4 expression was reduced by the introduction of mimic miR-29a in LNCaP cells. Luciferase activity from the construct harboring wild-type TRAF4 3'UTR was reduced by the mimic miR-29a and this reduction was diminished by introducing mutations at the predicted miR-29a binding site. On the other hand, TRAF4 was upregulated when transfected with the inhibitor of miR-29a in DU145 and PC3 cells. TRAF4 was significantly upregulated in patients with metastatic prostate cancer compared to those with localized prostate cancer. Furthermore, there was a significant inverse correlation between TRAF4 and miR-29a expression in tumor tissues from radical prostatectomy. Considered together, our results suggest that the tumor suppressor microRNA, miR-29a, is one
\end{abstract}

Correspondence to: Dr Takumi Shiraishi, The James Buchanan Brady Urological Institute, Department of Urology, The Johns Hopkins School of Medicine, 600 N Wolfe Street, 116 Marburg, Baltimore, MD 21287, USA

E-mail: tshirail@jhmi.edu

Key words: tumor necrosis factor receptor associated factor-4, microRNA-29a, metastatic prostate cancer of the regulators of TRAF4 expression in metastatic prostate cancer.

\section{Introduction}

The tumor necrosis factor receptor (TNFR)-associated factors (TRAFs) family of proteins consists of six members (TRAF1, TRAF2, TRAF3, TRAF4, TRAF5 and TRAF6) which act as major signal transducers for the TNFR superfamily as well as the interleukin-1 receptor/Toll-like receptor (IL-1R/TLR) superfamily (1). A common structural feature of TRAF proteins is a C-terminal TRAF domain (2) and TRAFs are associated with various biological functions including adaptive and innate immunity, embryonic development, stress response and bone metabolism $(1,3)$.

TRAF4 was originally identified as cysteine-rich motif associated with RING and TRAF domains (CART1) by differential screening of a cDNA library from lymph node metastasis of breast cancer (4). Unlike the other TRAF family proteins, the biological role of TRAF4 remains elusive, TRAF4 has been shown to be upregulated in ovarian, bladder, lung adenocarcinoma, small cell lung carcinoma, colon, breast cancers and prostate carcinomas by immunohistochemistry and TRAF4 gene amplification followed by TRAF4 overexpression was detected in $\sim 20 \%$ of the cases from six different types of carcinomas (5). However, the exact mechanism of TRAF4 overexpression in human cancers is still under investigation.

microRNAs (miRNAs) are small non-coding RNAs which negatively regulate gene expression at the post-transcriptional level and are involved in essential cellular functions such as proliferation, differentiation, cell cycle and apoptosis (6). Differential expression of miRNAs has been observed in several types of cancers and is shown to play an important role in cancer either as oncogenes or tumor suppressors (7-10). More specifically, it has been shown that microRNA-29a (miR-29a) is involved in apoptosis $(11,12)$, suggesting that its aberrant expression is closely related to cancer. Altered expression of miR-29 family has been observed in multiple cancers including cholangiocarcinoma, non-small lung cancer, 
nasopharyngeal cancer and acute myeloid leukemia (13-16). Furthermore, miR-29a has been reported to be downregulated in hepatocellular carcinoma and acute myeloid leukemia $(14,17)$. In prostate cancer, miR-29a has been shown to be downregulated only in castration-resistant prostate cancer compared to benign prostatic hyperplasia (18), indicating the possibility that miR-29a has a tumor suppressive function in prostate cancer.

Using the algorithm, TargetScan (19), we found that miR-29a is a putative miRNA that binds to the TRAF4 3' untranslated region (UTR). The aim of the present study was to investigate the expression of TRAF4 and miR-29a in localized and metastatic prostate cancer and examine whether TRAF4 expression is regulated by miR-29a in prostate cancer.

\section{Materials and methods}

Cells. The human prostate cancer cell lines (LNCaP, DU145 and PC3) were maintained in RPMI-1640 medium with $10 \%$ fetal bovine serum $(10 \% \mathrm{FBS})$ at $37^{\circ} \mathrm{C}$ in a humidified atmosphere containing $5 \% \mathrm{CO}_{2}$.

RNA extraction and quantitative real-time PCR for $m R N A$ and miRNA. Total RNA was isolated using the miRNeasy kit (Qiagen, Valencia, CA, USA). For mRNA detection, first strand cDNA was made from $1 \mu \mathrm{g}$ RNA using iScript cDNA synthesis kit (Bio-Rad Laboratories, Inc., Hercules, CA, USA) following the manufacturer's protocol in a total volume of $20 \mu \mathrm{l}$. Quantitative real-time PCR (Q-PCR) were carried out as previously described (20). In brief, the PCR reactions were performed with $0.2 \mu \mathrm{l}$ of cDNA template in $25 \mu \mathrm{l}$ of reaction mixture containing 12.5 $\mu \mathrm{l}$ of iQ SYBR-Green Supermix (Bio-Rad Laboratories) and $0.25 \mu \mathrm{mol} / 1$ each primer. PCR reactions were subjected to hot start at $95^{\circ} \mathrm{C}$ for $3 \mathrm{~min}$ followed by 45 cycles of denaturation at $95^{\circ} \mathrm{C}$ for $10 \mathrm{sec}$, annealing at $60^{\circ} \mathrm{C}$ for $30 \mathrm{sec}$ and extension at $72^{\circ} \mathrm{C}$ for $30 \mathrm{sec}$ using the CFX96 Real-Time PCR Detection System (Bio-Rad Laboratories). PCR primers were 5'-CCTGGTGCCTTTGAC AATCT-3' (forward) and 5'-CTCAGTGACGTGCTGTGG TT-3' (reverse) for TRAF4 and 5'-GAATATAATCCCAAGC GGTTTG-3' (forward) and 5'-ACTTCACATCACAGCTC CCC-3' (reverse) for TATA binding protein (TBP). TBP was used as an internal control.

For miRNA expression analysis, cDNA was synthesized from $10 \mathrm{ng}$ total RNA using TaqMan MicroRNA reverse transcription kit with miRNA specific RT primer (Applied Biosystems, Foster City, CA, USA) according to the manufacturer's protocol in a total volume of $15 \mu \mathrm{l}$. Q-PCR were carried out following the manufacturer's protocol. In brief, the PCR reactions were performed with $1.33 \mu \mathrm{l}$ of cDNA template in $20 \mu \mathrm{l}$ of reaction mixture containing $10 \mu \mathrm{l}$ of TaqMan $2 \mathrm{X}$ Universal PCR Master Mix, No AmpErase UNG (Applied Biosystems) and $1 \mu \mathrm{l}$ of TaqMan MicroRNA assays (20X). PCR reactions were subjected to hot start at $95^{\circ} \mathrm{C}$ for $10 \mathrm{~min}$ followed by 40 cycles of denaturation at $95^{\circ} \mathrm{C}$ for $15 \mathrm{sec}$ and annealing and extension at $60^{\circ} \mathrm{C}$ for $60 \mathrm{sec}$ using the CFX96 Real-Time PCR Detection System (Bio-Rad Laboratories). The assay names used in the present study were hsa-miR29a (\#000412) and RNU24 (\#001001) (Applied Biosystems). RNU24 was used as an internal control. Analysis and fold- differences were determined using the comparative threshold cycle method. All experiments were performed in triplicate and the data presented represents mean \pm SD.

Western blot analysis. Western blot analysis was performed as previously described (21). Briefly, cell lysates were prepared in whole cell lysis buffer (50 mM Tris- $\mathrm{HCl}$ pH 7.5, 1\% SDS) with $1 \mathrm{mM}$ dithiothreitol, $1 \mathrm{mM}$ phenylmethylsulfonyl fluoride and 1X Halt Protease Inhibitor Cocktail (Thermo Fisher Scientific, Rockford, IL, USA) followed by sonication and centrifugation $(14,000 \mathrm{rpm})$. Extracts were quantified using the Bio-Rad protein assay (Bio-Rad Laboratories). Lysates were subjected to SDS-PAGE and transferred to polyvinylidene difluoride membranes (Millipore, Bedford, MA, USA). Membranes were incubated with primary antibodies followed by horseradish peroxidase-conjugated secondary antibodies, and developed with the Super Signal West Dura Extended Duration Substrate kit (Thermo Fisher Scientific). Mouse monoclonal TRAF4 antibody (Origene, Rockville, MD, USA) was used as a primary antibody.

Mimic/inhibitor miRNA treatment. miRIDIAN microRNA mimics and hairpin inhibitors for hsa-miR-29a and controls were purchased from Thermo Fisher Scientific. One day prior to the transfection, cells were seeded without antibiotics at a density of $30-40 \%$. Cells were transiently transfected with each microRNA mimics and hairpin inhibitors $(50 \mathrm{nM})$ using DharmaFECT1 transfection reagent (Thermo Fisher Scientific) according to the manufacturer's protocol with some modification, in which the volume of DharmaFECT1 transfection reagent was reduced to half of the recommended volume to limit toxic effects.

Plasmids. To generate TRAF4 3'UTR reporter construct, the TRAF4 3'UTR region (1319 bp) was generated by PCR using PrimeSTAR MAX DNA polymerase (Takara, Shiga, Japan) from LNCaP genomic DNA. The primers used for amplification were the following: 5'-AATGCTAGCCATGACCTCAGT CAGGCACT-3' (forward) and 5'-ATACTCGAGAACAAATC TGGGAGGTGAGC-3' (reverse). The PCR product was digested with $\mathrm{NheI} / \mathrm{XhoI}$ and ligated into the same sites of pmirGLO Dual-luciferase miRNA target expression vector (Promega Corp., Madison, WI, USA) to produce pmir-TR4_ WT.

The construct, pmir-TR4_MT, which has mutations at the predicted miR-29a binding sites, were generated by site-directed mutagenesis using the Quick Change XL SiteDirected Mutagenesis kit (Stratagene, La Jolla, CA, USA) with the following oligonucleotides: sense (M1), 5'-CCTCAGGTGC CTCCAATTATGATTTCAGCCCTGGCCCCTG-3'; and antisense (M1), 5'-CAGGGGCCAGGGCTGAAATCATAA TTGGAGGCACCTGAGG-3'. Each sequence is identical to that of pmir-TR4_WT, except for the sequence in bold letters. All constructs were confirmed by sequencing.

Luciferase assay. The pmir-TR4_WT and the pmir-TR4_MT $(0.5 \mu \mathrm{g})$ were transfected into cells $\left(1 \times 10^{5}\right.$ cells/well in 24-well plates) using Lipofectamine LTX reagent (Invitrogen, Grand Island, NY, USA) with miRIDIAN microRNA mimics for hsa-miR-29a or non-targeting control (final $50 \mathrm{nM}$ ). After 

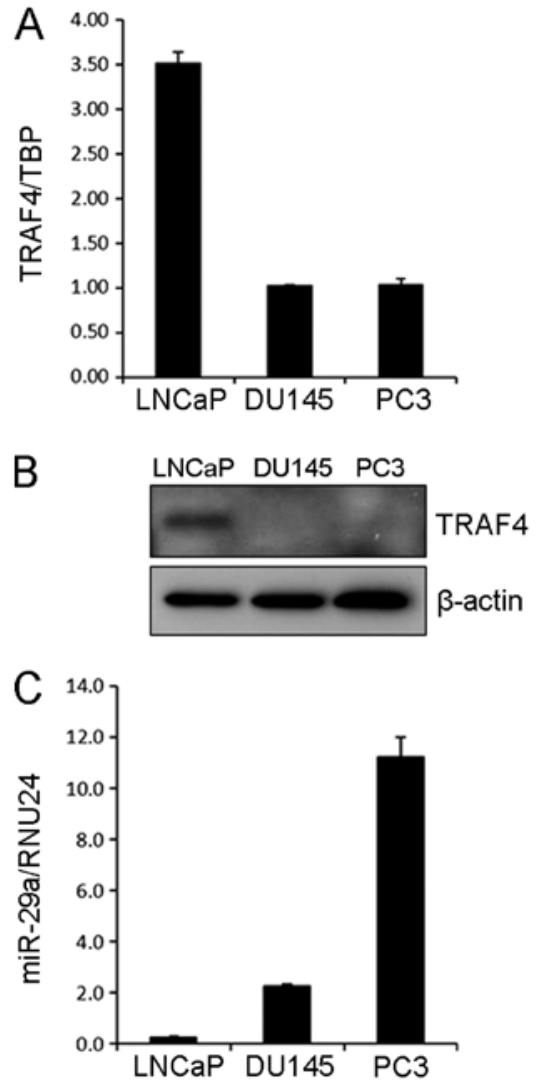

Figure 1. TRAF4 and miR-29a expression in prostate cancer cell lines. (A) TRAF4 mRNA expression was determined in LNCaP, DU145 and PC3 cells by Q-PCR. TRAF4 mRNA expression was normalized by TATA binding protein (TBP). The experiments were repeated three times. Data are presented as the mean of triplicate experiments \pm SD. (B) TRAF4 protein expression was detected by western blot analysis. $\beta$-actin was used as a loading control. (C) miR-29a expression was quantified in LNCaP, DU145 and PC3 cells by Q-PCR. The expression of miR-29a was normalized by RNU24. The experiments were repeated three times. Data are presented as the mean of triplicate experiments $\pm \mathrm{SD}$.

$48 \mathrm{~h}$, the cells were harvested using Reporter Lysis Buffer (Promega). The luciferase activity of the cell lysate was measured using the Dual-Glo luciferase reporter assay system (Promega) with FLUOstar Omega microplate reader (BMG Labtech, Ortenberg, Germany).

Clinical samples. Surgical specimens from 23 patients with clinically localized prostate cancer, ranging in age from 43 to 70 years (median 59 years) were collected and frozen at the time of radical prostatectomy at the Johns Hopkins Hospital. A preoperative serum PSA was a median of 7.59 (ng/ml) (range, 1.89-29.0). The Gleason score sum (GS) was: $6(n=15), 7(n=7)$ and $9(n=1)$, respectively. The use of surgical specimens for molecular analysis was approved by the Johns Hopkins Medicine Institutional Review Boards.

In other experiments, samples from clinically localized prostate cancer $(n=20)$ and soft tissue metastasis $(n=20)$ were obtained at University of Washington. The age range of the patients with clinically localized prostate cancer was 48-75 years (median, 58 years) and a preoperative serum PSA was a median of $7.54(\mathrm{ng} / \mathrm{ml})$ (range, 2.4-64.0). The GS was: $6(\mathrm{n}=3)$, $7(n=14), 8(n=1)$ and $9(n=2)$, respectively. Soft tissue metastasis was obtained from lymph node $(\mathrm{n}=8)$, liver $(\mathrm{n}=5)$, adrenal

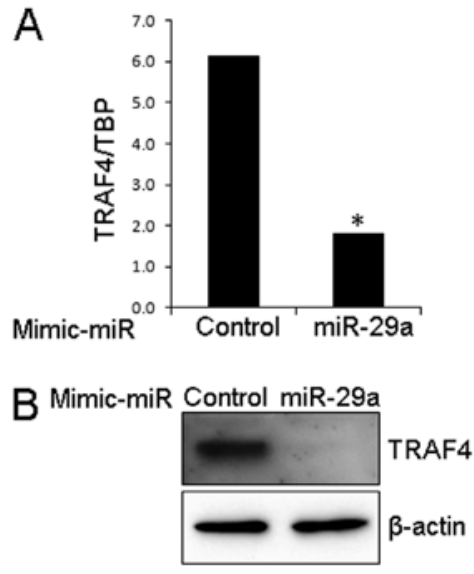

Figure 2. Effect of mimic miR-29a on TRAF4 expression in LNCaP cells (A) LNCaP cells were transiently transfected with mimic miRNA for miR-29a and control and harvested $72 \mathrm{~h}$ after transfection. TRAF4 mRNA levels were determined by Q-PCR. TRAF4 mRNA expression was normalized by TATA binding protein (TBP). The experiments were repeated three times. Data are presented as the mean of triplicate experiments $\pm \mathrm{SD}$. $\left({ }^{*} \mathrm{P}<0.01\right.$, Student's t-test). (B) LNCaP cells were transiently transfected with mimic miRNA for miR-29a and control and harvested $96 \mathrm{~h}$ after transfection. TRAF4 protein expression was detected by western blot analysis. $\beta$-actin was used as a loading control.

$(\mathrm{n}=1)$, bladder $(\mathrm{n}=1)$, kidney $(\mathrm{n}=1)$, lung $(\mathrm{n}=1)$ and pancreas $(n=1)$, respectively. The specimens were used with the approval of the University of Washington Institutional Review Boards.

Statistical analysis. Data are presented as the mean \pm SD and statistical differences between two groups of data were analyzed using the Student's t-test. TRAF4 expression levels in each patient group were compared by the Mann-Whitney $\mathrm{U}$ test. Correlation between TRAF4 and miR-29a expression were analyzed using the Pearson's correlation coefficient test. Statistical significance was applied to P-values of $<0.05$.

\section{Results}

Expression of TRAF4 and miR-29a in prostate cancer cells. We first analyzed the TRAF4 expression levels in three different PCa cell lines (LNCaP, DU145 and PC3 cells). TRAF4 was highly expressed in LNCaP cells compared to DU145 and PC3 cells at both mRNA and protein levels (Fig. 1A and B). Since miR-29a was predicted to bind TRAF4 3'UTR by the algorithm, TargetScan (19), we examined the expression of miR-29a in these cell lines. As shown in Fig. 1C, high and moderate expression levels of miR-29a were observed in PC3 and DU145 cells, respectively while LNCaP cells exhibited the lowest expression of miR-29a among these cell lines.

Effect of mimic miR-29a on TRAF4 expression in LNCaP cells. Since we observed an inverse association between TRAF4 and miR-29a expression in PCa cell lines, we next investigated the effect of mimic miR-29a on TRAF4 expression in LNCaP cells in which miR-29a was downregulated and endogenous TRAF4 was expressed. When cells were treated with mimic miR-29a, the expression of TRAF4 was significantly reduced at both mRNA and protein levels in LNCaP cells (Fig. 2). 

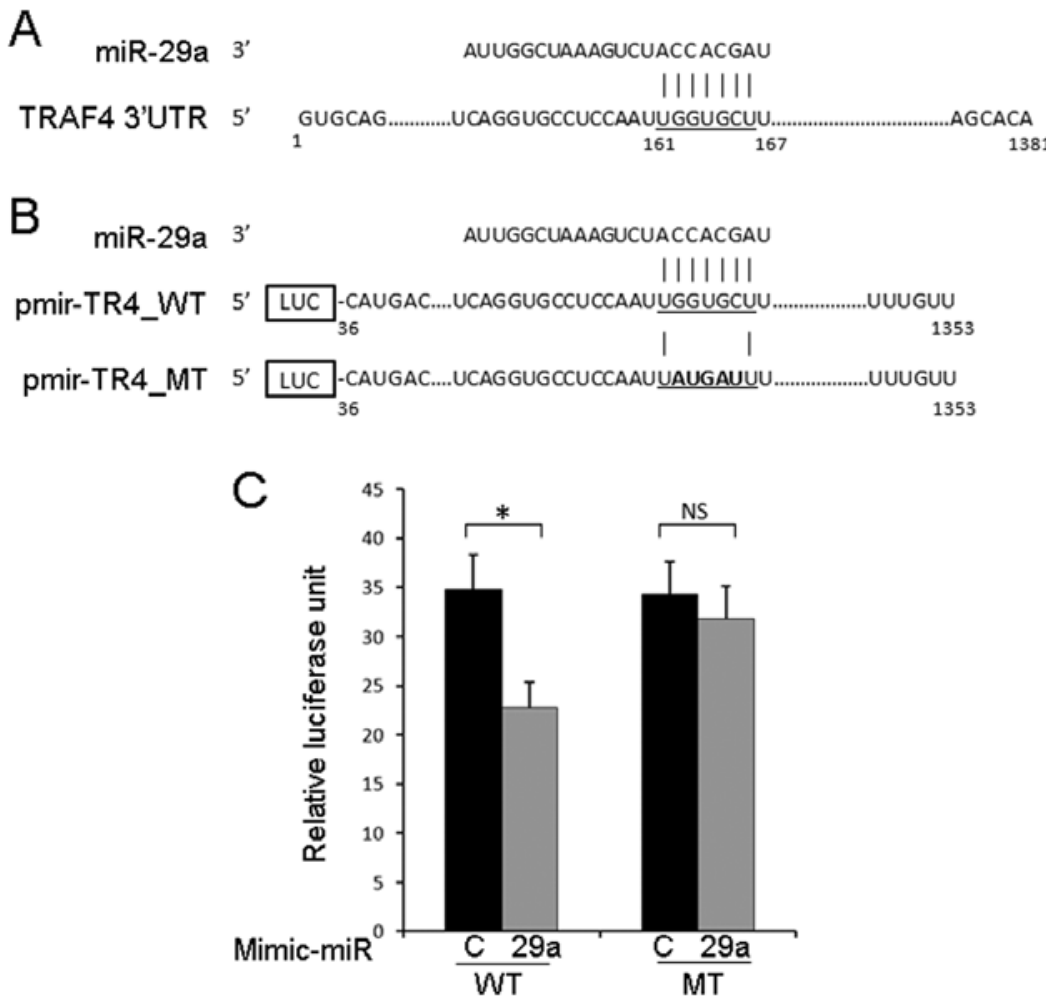

Figure 3. miR-29a targets the TRAF4 3'UTR. (A) Predicted target site of miR-29a at the TRAF4 3'UTR by TargetScan. (B) Schematic description of the TRAF4 3'UTR construct (pmir-TR4_WT and pmir-TR4_MT). (C) Luciferase activity was measured in transiently transfected LNCaP cells treated with mimic miR-29a (29a) and control (C) miRNA for $48 \mathrm{~h}$. The experiments were repeated three times. Data were presented as the mean of triplicate experiments \pm SD. ('P<0.01, Student's t-test; NS, not significant).
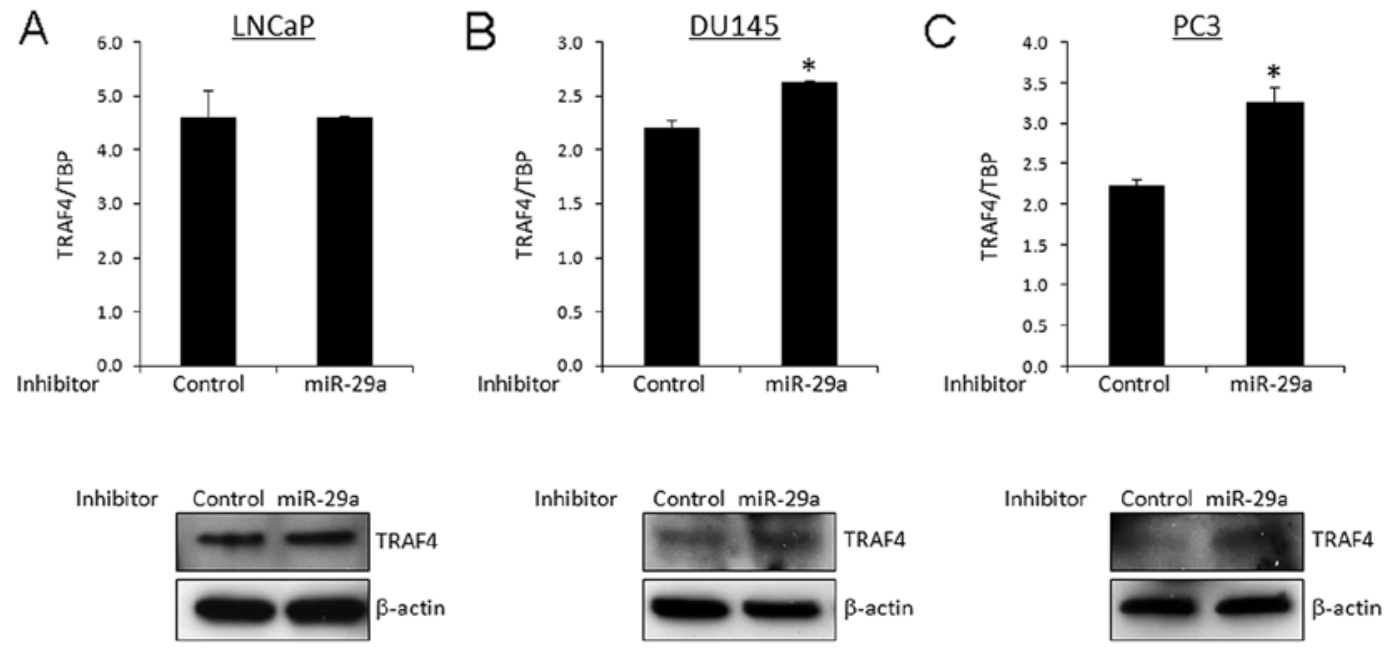

Figure 4. Effect of inhibitor miR-29a on TRAF4 expression in prostate cancer cells. (A) LNCaP, (B) DU145 and (C) PC3 cells were treated with inhibitor of miR-29a and control for $96 \mathrm{~h}$. TRAF4 mRNA levels were determined by Q-PCR (upper panel). TRAF4 protein expression was detected by western blot analysis (lower panel). TRAF4 mRNA expression was normalized by TATA binding protein (TBP). $\beta$-actin was used as a loading control for western blot analysis. The experiments were repeated three times. Data are presented as the mean of triplicate experiments \pm SD. ("P<0.01, Student's t-test).

TRAF4 3'UTR is a direct target of miR-29a. To investigate whether miR-29a binds to the TRAF4 3'UTR, we generated a reporter construct harboring a 1319-bp fragment of the TRAF4 3'UTR downstream of the firefly luciferase gene (Fig. 3A and B). As shown in Fig. 3C, luciferase activity from pmir-TR4_WT was significantly reduced when mimic miR-29a was co-transfected, and this suppressive effect of miR-29a was attenuated by the introduction of mutation at the predicted miR-29a binding site. These results suggested that miR-29a inhibits the expression of TRAF4 through the direct binding to the TRAF4 3 'UTR.

Effect of inhibitor miR-29a on TRAF4 expression in prostate cancer cells. To confirm the suppressive effect of TRAF4 expression by miR-29a, we treated prostate cancer cells with inhibitor miR-29a. In DU145 and PC3 cells expressing 

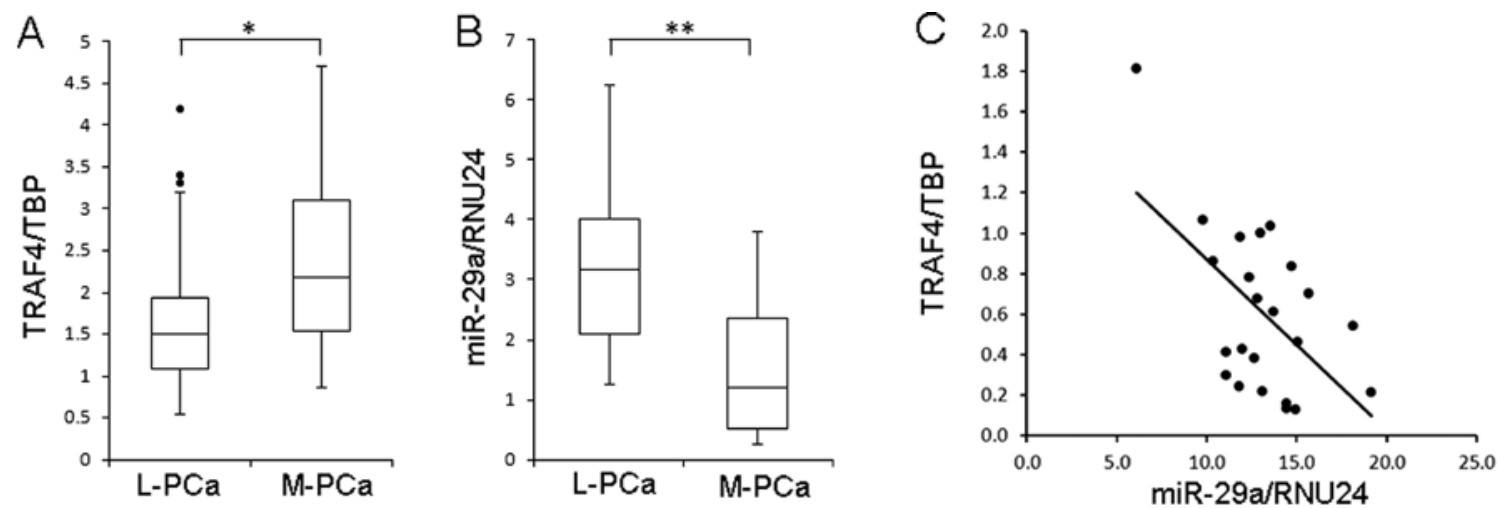

Figure 5. TRAF4 expression is inversely correlated with miR-29a in prostate cancer. (A) TRAF4 mRNA expression was determined in localized (L-PCa) $(n=20)$ and metastatic (M-PCa) $(n=20)$ prostate cancer by Q-PCR. TRAF4 mRNA expression was normalized by TATA binding protein (TBP). Patient characteristics are described in the Materials and methods. ("P<0.05, Mann-Whitney U test). (B) The expression of miR-29a was determined in localized (L-PCa) $(n=20)$ and metastatic $(M-P C a)(n=20)$ prostate cancer by Q-PCR. The expression of miR-29a was normalized by RNU24. Patient characteristics are described in the Materials and methods. ( ${ }^{* *} \mathrm{P}<0.01$, Mann-Whitney U test). (C) TRAF4 and miR-29a expression was quantified in clinically localized prostate cancer $(n=23)$. Correlation coefficient between TRAF4 and miR-29a expression was analyzed using the Pearson's correlation coefficient test $(n=23, r=-0.56, P<0.01)$.

moderate to high levels of miR-29a, the treatment with inhibitor miR-29a increased the expression of TRAF4 at both mRNA and protein levels (Fig. 4B and C). On the other hand, no obvious effect was observed by the introduction of inhibitor miR-29a in LNCaP cells in which the expression level of miR-29a was low compared to DU145 and PC3 cells (Fig. 4A).

Inverse correlation between TRAF4 and miR-29a expression in patients with prostate cancer. We next examined the expression of TRAF4 and miR-29a in prostate tumor samples from radical prostatectomy $(n=20)$ and soft tissue metastasis $(n=20)$. As shown in Fig. 5A, the expression of TRAF4 was significantly higher in samples from metastatic prostate cancer compared to localized prostate cancer. On the other hand, miR-29a expression was significantly lower in metastatic prostate cancer than in localized prostate cancer (Fig. 5B). Furthermore, there was a significant inverse correlation between TRAF4 and miR-29a expression in localized prostate cancer $(\mathrm{n}=23)$.

\section{Discussion}

In the present study, we demonstrated that TRAF4 is upregulated and miR-29a is downregulated in metastatic prostate cancer and TRAF4 is a direct target of miR-29a. These results might provide a clue to elucidate the molecular mechanism of prostate cancer progression.

TRAF4 is a unique member of TRAF family proteins which are known to be involved in immunity, inflammation and apoptosis (1). However, TRAF4 appears to be a distinct member of the TRAF family, and its functional role, especially in cancer, remains under investigation (22). It was reported that TRAF4 is a positive regulator of bone morphogenetic proteins (BMP) (23). BMPs are members of the transforming growth factor- $\beta$ (TGF- $\beta$ ) superfamily and play important roles in embryogenesis and organogenesis. Since BMPs are well known to be involved in the development of bone metastasis in prostate cancer (24), overexpression of TRAF4 might play an important role in the bone metastasis by activating
BMP signaling in prostate cancer. Bone is the most common metastatic site in patients with prostate cancer (25) and the complications from bone metastasis are a major problem when treating patients with prostate cancer. Thus, TRAF4 may represent a novel therapeutic target to treat patients with bone metastasis, and further experiments are required to investigate the role of TRAF4 in the development of bone metastasis.

The exact role of miR-29a in cancer still remains elusive. However, accumulating evidence suggests that miR-29a mainly acts as a tumor suppressor by modulating apoptosis. Interestingly, it has been shown that the expression of miR-29a is repressed by c-Myc, Hedgehog and NF- $\mathrm{BB}$ at the transcriptional level $(15,26)$. The oncogenic role of c-Myc in prostate cancer has been well studied (27) and it is known that c-Myc is highly upregulated in castration-resistant prostate cancer $(28,29)$. Thus, overexpression of TRAF4 through the downregulation of miR-29a by c-Myc might be one of the mechanisms in prostate cancer progression and contribute to the development of bone metastasis.

Nuclear factor- $\kappa \mathrm{B}(\mathrm{NF}-\kappa \mathrm{B})$ proteins are an important class of transcriptional regulators that sustain the malignant phenotype in inflammation-related cancer (30). In prostate cancer, the NF- $\mathrm{KB}$ activity is reported to be higher in metastatic prostate cancer than in localized disease (31), and elevated activity of NF- $\kappa \mathrm{B}$ is correlated with a poor prognosis in primary prostate cancer $(32,33)$. Furthermore, significant deregulation of the NF- $\kappa \mathrm{B}$ pathway was identified in metastatic prostate cancer (34). It has been shown that TRAF4 activates NF- $\kappa B$ through the glucocorticoid-induced TNF-R (GITR) (35). Considering the suppressive effect of NF- $\mathrm{kB}$ on miR-29a transcription (15), there might be a positive feedback loop from NF- $\kappa$ B to TRAF4 via miR-29a, leading to the progression of prostate cancer.

In summary, we have demonstrated that TRAF4 is overexpressed and miR-29a is downregulated in metastatic prostate cancer. Furthermore, we clearly showed that miR-29a is a negative regulator of TRAF4 through the direct binding at the TRAF4 3'UTR. Although further studies are required to elucidate the functional role of TRAF4, these findings 
will shed new light on the molecular mechanism of prostate cancer progression and may provide additional insight to develop a novel therapeutic strategy to manage patients with metastatic prostate cancer.

\section{Acknowledgements}

The authors wish to thank the members of the Kulkarni Laboratory for many helpful discussions.

\section{References}

1. Chung JY, Park YC, Ye H and Wu H: All TRAFs are not created equal: common and distinct molecular mechanisms of TRAFmediated signal transduction. J Cell Sci 115: 679-688, 2002.

2. Rothe M, Wong SC, Henzel WJ and Goeddel DV: A novel family of putative signal transducers associated with the cytoplasmic domain of the $75 \mathrm{kDa}$ tumor necrosis factor receptor. Cell 78: 681-692, 1994

3. Xie P: TRAF molecules in cell signaling and in human diseases. J Mol Signal 8: 7, 2013.

4. Regnier CH, Tomasetto C, Moog-Lutz C, et al: Presence of a new conserved domain in CART1, a novel member of the tumor necrosis factor receptor-associated protein family, which is expressed in breast carcinoma. J Biol Chem 270: 25715-25721, 1995.

5. Camilleri-Broet S, Cremer I, Marmey B, et al: TRAF4 overexpression is a common characteristic of human carcinomas. Oncogene 26: 142-147, 2007.

6. Bartel DP: MicroRNAs: genomics, biogenesis, mechanism, and function. Cell 116: 281-297, 2004.

7. Dews M, Homayouni A, Yu D, et al: Augmentation of tumor angiogenesis by a Myc-activated microRNA cluster. Nat Genet 38: 1060-1065, 2006.

8. El Baroudi M, Cora D, Bosia C, Osella M and Caselle M: A curated database of miRNA mediated feed-forward loops involving MYC as master regulator. PLoS One 6: e14742, 2011.

9. Hermeking H: p53 enters the microRNA world. Cancer Cell 12 414-418, 2007.

10. Hermeking H: The miR-34 family in cancer and apoptosis. Cell Death Differ 17: 193-199, 2010.

11. Kole AJ, Swahari V, Hammond SM and Deshmukh M: miR-29b is activated during neuronal maturation and targets $\mathrm{BH} 3$-only genes to restrict apoptosis. Genes Dev 25: 125-130, 2011.

12. Park SY, Lee JH, Ha M, Nam JW and Kim VN: miR-29 miRNAs activate $p 53$ by targeting $p 85 \alpha$ and CDC42. Nat Struct Mol Biol 16: 23-29, 2009.

13. Fabbri M, Garzon R, Cimmino A, et al: MicroRNA-29 family reverts aberrant methylation in lung cancer by targeting DNA methyltransferases $3 \mathrm{~A}$ and 3B. Proc Natl Acad Sci USA 104: 15805-15810, 2007.

14. Garzon R, Heaphy CE, Havelange V, et al: MicroRNA 29b functions in acute myeloid leukemia. Blood 114: 5331-5341, 2009.

15. Mott JL, Kobayashi S, Bronk SF and Gores GJ: mir-29 regulates Mcl-1 protein expression and apoptosis. Oncogene 26: 6133-6140, 2007.

16. Sengupta S, den Boon JA, Chen IH, et al: MicroRNA 29c is down-regulated in nasopharyngeal carcinomas, up-regulating mRNAs encoding extracellular matrix proteins. Proc Natl Acad Sci USA 105: 5874-5878, 2008.
17. Xiong Y, Fang JH, Yun JP, et al: Effects of microRNA-29 on apoptosis, tumorigenicity, and prognosis of hepatocellular carcinoma. Hepatology 51: 836-845, 2010.

18. Porkka KP, Pfeiffer MJ, Waltering KK, Vessella RL, Tammela TL and Visakorpi T: MicroRNA expression profiling in prostate cancer. Cancer Res 67: 6130-6135, 2007.

19. Lewis BP, Shih IH, Jones-Rhoades MW, Bartel DP and Burge CB: Prediction of mammalian microRNA targets. Cell 115: 787-798, 2003.

20. Shiraishi T, Terada N, Zeng Y, et al: Cancer/Testis Antigens as potential predictors of biochemical recurrence of prostate cancer following radical prostatectomy. J Transl Med 9: 153, 2011.

21. Yoshida T, Shiraishi T, Horinaka M, et al: Lipoxygenase inhibitors induce death receptor 5/TRAIL-R2 expression and sensitize malignant tumor cells to TRAIL-induced apoptosis. Cancer Sci 98: 1417-1423, 2007.

22. Kedinger $\mathrm{V}$ and Rio MC: TRAF4, the unique family member. Adv Exp Med Biol 597: 60-71, 2007.

23. Kalkan T, Iwasaki Y, Park CY and Thomsen GH: Tumor necrosis factor-receptor-associated factor- 4 is a positive regulator of transforming growth factor- $\beta$ signaling that affects neural crest formation. Mol Biol Cell 20: 3436-3450, 2009.

24. Ye L, Lewis-Russell JM, Kyanaston HG and Jiang WG: Bone morphogenetic proteins and their receptor signaling in prostate cancer. Histol Histopathol 22: 1129-1147, 2007.

25. Bubendorf L, Schopfer A, Wagner U, et al: Metastatic patterns of prostate cancer: an autopsy study of 1,589 patients. Hum Pathol 31: 578-583, 2000

26. Chang TC, Yu D, Lee YS, et al: Widespread microRNA repression by Myc contributes to tumorigenesis. Nat Genet 40: 43-50, 2008.

27. Ellwood-Yen K, Graeber TG, Wongvipat J, et al: Myc-driven murine prostate cancer shares molecular features with human prostate tumors. Cancer Cell 4: 223-238, 2003.

28. Hawksworth D, Ravindranath L, Chen Y, et al: Overexpression of C-MYC oncogene in prostate cancer predicts biochemical recurrence. Prostate Cancer Prostatic Dis 13: 311-315, 2010.

29. Jenkins RB, Qian J, Lieber MM and Bostwick DG: Detection of c-myc oncogene amplification and chromosomal anomalies in metastatic prostatic carcinoma by fluorescence in situ hybridization. Cancer Res 57: 524-531, 1997.

30. Karin M: Nuclear factor- $\mathrm{B}$ in cancer development and progression. Nature 441: 431-436, 2006.

31. Ismail HA, Lessard L, Mes-Masson AM and Saad F: Expression of NF- $\kappa$ B in prostate cancer lymph node metastases. Prostate 58: 308-313, 2004.

32. Lessard L, Begin LR, Gleave ME, Mes-Masson AM and Saad F: Nuclear localisation of nuclear factor-kappaB transcription factors in prostate cancer: an immunohistochemical study. Br J Cancer 93: 1019-1023, 2005.

33. Lessard L, Karakiewicz PI, Bellon-Gagnon P, et al: Nuclear localization of nuclear factor-kappaB p65 in primary prostate tumors is highly predictive of pelvic lymph node metastases. Clin Cancer Res 12: 5741-5745, 2006.

34. Setlur SR, Royce TE, Sboner A, et al: Integrative microarray analysis of pathways dysregulated in metastatic prostate cancer. Cancer Res 67: 10296-10303, 2007.

35. Esparza EM and Arch RH: TRAF4 functions as an intermediate of GITR-induced NF-kappaB activation. Cell Mol Life Sci 61: 3087-3092, 2004. 